\title{
Blepharolifting without Surgery
}

\author{
Fabrizio Bonucci* \\ Department of Dentistry, Freelance for Non-Invasive Medicine Esthetic Dentistry in Rome, Italy
}

*Corresponding author: Fabrizio Bonucci, Department of Dentistry, Freelance for NonInvasive Medicine Esthetic Dentistry in Rome, Italy.

Received Date: July 15, 2019

Published Date: July 22, 2019

\section{Opinion}

Looking for the "Beauty" has always played a fundamental role in the choices of every moment of our lives. Humankind has always tried to delay the negative effects of time, which inexorably destroys the Beauty. To achieve this goal, we inevitably relied on randomness and on the genetically predetermined statistics of Nature and, therefore, we were looking for male and female subjects simply as younger as possible, because very little could be done from the medical-surgical point of view, it was, in fact, more with Cosmetics that we could delay these negative effects.

Today, an increasing number of people are asking Aesthetic Medicine for less invasive solutions to improve their appearance rather than to heal more or less serious diseases. This is due to the fact that, Beauty-Perception is not an absolute factor but relative to the environment in which we live. The concept of "beautiful" is different according to the culture, the tradition and the historical moment of reference. Therefore, more a person is far from the Golden-Ratio, more is perceived as not "attractive" if not even different, negative and dangerous.

We are social animals and to communicate we use a non-verbal language too and therefore the first contact we have is visual through our eyes, so having a look not "tired" but "young and healthy" becomes the main target of many people. In fact, one of the most requested "aesthetic" operation all over the world is surgical blepharoplasty.

A lot of specialists all over the world are able to achieve excellent outcomes with high success rates, but because, unfortunately, you must to take in a big consideration side-effects too, statistically always possible, but unacceptable if you consider the purely "aesthetic" feature of the treatments, many, perhaps too many, possible patients do not even consider this solution, sometime also due to the high cost, connected with the use of an operating room and a full staff of several specialists. Therefore, for a modern, skilled and up-to-date surgeon, the ideal would be to use an easy, safe, economical, uncomplicated procedure, without general anesthesia, without sutures and with the fastest and most natural healing as possible.

Many devices were recommended to try to achieve a result like this, but each one showed later other limitations or complications. In the last years in Aesthetic Medicine a new type of devices above all is proving to be the Gold Standard for several treatments not only purely aesthetic the Cold Atmospheric Plasma, term by which we call the plasmas artificially produced at atmospheric pressure and temperature. Plasma is the Fourth State of Matter and is present in Nature in various forms such as the sun, stars, nebulae, the solar crown, solar winds, the ionosphere, the northern lights and lightning.

Artificial Plasma, a phenomenon visible to our eyes through a small lightning bolt, instead, is an ionized gas, composed of charged particles (electrons, ions), atoms and excited molecules, radicals and UV photons, ROS (Reactive Oxygen Species) and RNS (Reactive Nitrogen Species), produced within the space of about 1 $\mathrm{mm}$ between two electrodes (the instrument and the skin) when the sudden addition of a large amount of energy at a very small point and time causes the ripping of the electrons from the atoms and molecules and their instantaneous disappearance, called "sublimation" i.e. the transition from the solid state directly to the gaseous state by skipping the liquid state. With this phenomenon it is possible, therefore, to eliminate many blemishes without surgery, often without anesthesia, always without stitches and for this reason they have opened a new way to perform Aesthetic Medicine defined as Non-invasive and Non-Surgical, i.e. no bloody, without surgery, without scalpel, without damage to the surrounding tissues, with a very selective and extremely precise action and with healings unthinkable with any other instrument.

Many histological studies have shown that not only with the Plasma device we have the sublimation of the upper skin layer 
without exceeding the basal membrane, without touching the blood capillaries, hair follicles and melanocytes, but that we also have the creation of collagen type III. In fact, thanks to the safety, softness, precision, reliability, speed and, therefore, economy features of the Plasma Device, more and more patients appreciate this technological innovation and ask to use this procedure to obtain a natural, dynamic and interactive improvement of their appearance, not only of the eyelid.

A particular aspect not to be underestimated, moreover, is that, with classical surgery, the specialist must trust what he has drawn on the patient's eyelids before the surgery, because, due to the necessary local anesthesia too, the tissues swell and stretch, losing many anatomical references taken into account during the surgical planning phase. With the plasma device, on the other hand, not only is local anesthesia in cream, occluded on the eyelids for about 45 minutes before, but the procedure is also dynamic and interactive, because, by asking the patient to move his eyes and squeezing his eyelids between our fingers, you can easily see live what we are doing and where we need to eliminate the excess tissue with a result, instantaneous, natural and lasting over time.
In the following 7-10 days we only need to provide a simple local and systemic antiphlogistic protocol, so much so that the return to social and working life is considerably faster than that resulting from a classic surgery.

Finally, we must consider that many corrections of some less successful surgeries can benefit greatly from this non-invasive procedure without having to use general anesthesia again in the operating room. Therefore, the intrinsic safety, the low learning curve, the natural and long-lasting result, the rapid Return-OfInvestment and the growing success all over the world, make this procedure so interesting not only from a clinical point of view but above all also from the point of view of marketing and promotion for the specialist who will want to combine it with the classical techniques.

\section{Acknowledgment}

None.

\section{Conflicts of Interest}

No conflicts of interest. 